\title{
Automatic assignment of labels in Topic Modelling for Russian Corpora
}

Aliya Mirzagitova, Olga Mitrofanova

Department of Mathematical Linguistics, St. Petersburg State University, Russia

https://doi.org/10.36505/ExLing-2016/07/0025/000284

\begin{abstract}
The main goal of this paper was to improve topic modelling algorithms by introducing automatic topic labelling, a procedure which chooses a label for a cluster of words in a topic. Topic modelling is a widely used statistical technique which allows to reveal internal conceptual organization of text corpora. We have chosen an unsupervised graph-based method and elaborated it with regard to Russian. The proposed algorithm consists of two stages: candidate generation by means of PageRank and morphological filters, and candidate ranking. Our experiments on a corpus of encyclopedic texts on linguistics has shown the advantages of labelled topic models for NLP applications.

Key words: topic modelling, topic labelling, Russian corpora.
\end{abstract}

\section{Introduction}

In recent years, topic modelling has become one of the most fruitful statistical NLP procedures which allows to reveal internal conceptual organization of text corpora. A topic model is constituted by a family of probability distributions over a set of topics extracted from a corpus, a set of words occurring in a corpus and a set of texts forming a corpus. Various algorithms of topic modelling (LSA, pLSA, LDA etc.) have been successfully applied to English corpora (Daud et al. 2010) in research dealing with information retrieval, content analysis, WSD, machine translation, etc. However, Russian corpora are seldom involved in topic modelling procedures. Certain positive results have been described in (Mitrofanova 2015). Our project tries to fill in this gap.

Resulting topics are commonly represented as the top $n$ terms with the highest probabilities, which often poses a great challenge in their proper and accurate interpretation. Assignment of a topic labels, i.e. a single word or a phrase able to describe the semantics of a given topic, significantly assists in this task. In most of the works, topic labelling is conducted manually, which is a tedious process prone to subjectivity.

There have been proposed numerous techniques of automatic topic labelling for English texts, including those relying only on the content of a given corpus (Mei et al. 2007), and those requiring external resources like Wikipedia (Lau et al. 2011) or various ready-made ontologies. All of them are two-stage methods varying in the means of generating and

ExLing 2016: Proceedings of $7^{\text {th }}$ Tutorial and Research Workshop on Experimental Linguistics, 27 June - 2 July 2016, Saint Petersburg, Russia 
ranking candidate labels. In this paper, we adopt the unsupervised graphbased approach as described in (Aletras, Stevenson 2014). We elaborate it to make it applicable for Russian corpora of specialised texts by modifying it at both stages.

\section{Methodology \\ Candidate generation}

In order to generate candidate labels, the first 10 topic words are used to query a search engine. After that, the titles of the top 30 search results are combined into a text, which is then tokenised and lemmatised. Subsequently, an oriented text graph $G=\{V, E\}$ is created, where $V$ is a set of nodes containing lemmas, $E$ is a set of edges. Two nodes are connected if the respective lemmas occur in the window of \pm 2 words. We experimented with three approaches to the weighting of the graph.

I. All of the edges are equal to 1 (unweighted graph).

II. The edges are weighted according to the co-occurrence frequency for corresponding lemmas calculated inside the given text.

III. The edges are weighted with PMI values computed using the Russian Wikipedia as a referential corpus (228 million tokens).

Next, the PageRank value (Mihalcea 2004) is computed for each node. The obtained text graph now takes the following form: more important words have larger nodes with higher PageRank values, while more semantically related bigrams have thicker edges with bigger weight.

Since Wikipedia does not have individual articles for most technical terms, we cannot verify the validity of a candidate label by checking whether it is a title, as it was proposed in the previous approaches. Therefore, appropriate n-grams are filtered from the text graph according to the following morphological patterns: Adj $+\mathrm{N}, \mathrm{N}+\mathrm{N}$ in genitive case, $\mathrm{N}+$ Prep $+\mathrm{N}, \mathrm{N}+$ Conj $+\mathrm{N}$, etc. The contact phrases are concatenated into a single group and added as a supplementary candidate label.

\section{Candidate ranking}

The second stage includes ranking of the extracted candidates. We examine the next three possible ranking metrics for each phrase label.

A. Simply summing the scores of the constituent words.

B. Normalizing the sum of the scores with regard to the phrase length.

C. Multiplying the sum by the coefficient calculated as $1+1 / i$, where $i$ is the position of the topic word in the original query. Thus we use the information about the probability of a constituent word belonging to the topic. 


\section{Experimental evaluation}

For experiments, we collected a corpus of Russian encyclopaedic texts on linguistics containing of 1,900 documents with a total of 1,3 million tokens. After pre-processing, that is lemmatising with an open-source tool pymorphy 2 and removing stop words, the size of the experimental corpus reduced to 800,501 tokens.

We performed a series of experiments on topic modelling with LDA algorithm implemented within a scikit-learn package for Python and obtained 20 topics, i.e. non-structured clusters of semantically related words. Finally, we automatically assigned a label to each topic.

\section{Evaluation and results}

To evaluate the quality of the automatic assignment, we asked experts to manually assess the extracted labels according to the following ordinal scale from 0 to 3 as suggested in (Lau et al. 2011):

0 Label is completely irrelevant for the topic.

1 Label is hardly related to the topic and/or it is ungrammatical.

2 Label is semantically related to the topic, but covers its content only partially and/or has grammatical mistakes.

3 Label perfectly describes the topic and it is grammatically correct.

In addition, we had to consider the grammaticality of the labels in case of erroneously extracted phrases by means of morphological patterns discussed earlier.

We used choosing the first topic word with the highest marginal probability as a baseline method. The results for each experimental configuration and the baseline are reported in Table 1.

Table 1. Evaluation results for each experimental configuration.

\begin{tabular}{|c|c|c|c|c|}
\hline \hline \multirow{2}{*}{ Label ranking } & \multicolumn{3}{|c|}{ Graph weighting } & \multirow{2}{*}{ Baseline } \\
\cline { 2 - 4 } & I & II & III & \\
\hline A & 2.01 & 2.03 & 2.07 & \multirow{2}{*}{1.03} \\
\hline B & 1.63 & 1.70 & 1.87 & \\
\hline C & 1.70 & 1.73 & 1.77 & \\
\hline \hline
\end{tabular}

\section{Discussion}

In this study, we address the gap in topic modelling for Russian corpora and present an algorithm for automatic assignment of topic labels adapted for Russian. It is based on the method described in (Aletras, Stevenson 2014), but differs from it in several respects. In particular, we introduced a step of identifying valid phrases using morphological patterns. Moreover, we conducted a number of experiments with various 
combinations of procedures for weighting a text graph and ranking the candidate labels.

The expert evaluation of results indicates that building a graph weighted with PMI values and ranking the candidates by simply summing the PageRank scores of the constituent words (A) performs best (2.07 out of 3). However, using inner co-occurrence frequency instead of PMI has also shown acceptable results (2.03), which means that it is not necessary to perform the heavy computations of association scores on a referential corpus.

The lower results for candidate ranking using normalised sum (B) and a special coefficient reflecting the importance of a topic word from a query $(\mathrm{C})$ can be explained by the bias of these metrics. Phrases extracted by the $\mathrm{B}$ methods tend to be short and too general, whereas $\mathrm{C}$ favors all the candidates with a topic word while ignoring other relevant labels.

Future work could include improvement of the phrase extraction algorithm, e.g. instead of filtering n-grams with part-of-speech patterns we could apply shallow parsing and consider syntactic chunks as candidate labels.

\section{References}

Aletras N., Stevenson M., Court R. 2014. Labelling Topics using Unsupervised Graphbased Methods. In Proc. of the 52nd Annual Meeting of the Association for Computational Linguistics, vol. 2, 631-636, Baltimore, USA.

Daud A., Li J., Zhou L., Muhammad F. 2010. Knowledge discovery through directed probabilistic topic models: a survey. Frontiers of Computer Science in China 4, 280 301.

Lau J., Grieser K., Newman D., Baldwin T. 2011. Automatic Labelling of Topic Models. In Proc. of the 49th Annual Meeting of the Association for Computational Linguistics: Human Language Technologies, vol. 1, 1536-1545, Stroudsburg, USA.

Mei Q., Shen X., Zhai C. 2007. Automatic labeling of multinomial topic models. In Proc. of the 13th Intern. Conference on Knowledge discovery and data mining, 490, New York, USA.

Mihalcea R. 2004. TextRank: Bringing Order into Texts. In Proc. of EMNLP 2004, 404-411, Barcelona, Spain.

Mitrofanova, O.A. 2015. Verojatnostnoje modelirovanije tematiki russkojazychnyh korpusov tekstov s ispol'zovanijem kompjuternogo instrumenta GenSim. [Probabilistic topic modeling of the Russian text corpora by means of GenSim toolkit]. In Trudy mezhdunarodnoj konferencii «Korpusnaja lingvistika - 2015», St.Petersburg, Russia. 